\title{
Superconductivity in Mutual Chern-Simons Gauge Theory
}

\author{
Peng Ye*, Long Zhang, and Zheng-Yu Weng \\ Institute for Advanced Study, Tsinghua University, Beijing, 100084, People's Republic of China
}

(Dated: March 23, 2022)

\begin{abstract}
In this work, we present a topological characterization of superconductivity in a prototype electron fractionalization model for doped Mott insulators. In this model, spinons and holons are coupled via the mutual Chern-Simons gauge fields. We obtain a low-lying effective description of the collective current fluctuations by integrating out the matter fields, which replaces the conventional GinzburgLandau action to describe the generalized rigidity of superconductivity. The superconducting phase coherence is essentially characterized by a topological order parameter related to a Gaussian linking number, and an experiment is proposed to probe this topological property. We further show that a gauge-neutral fermionic mode can naturally emerge in this model, which behaves like a Bogoliubov quasiparticle.

PACS numbers: $74.40 . \mathrm{Kb}, 74.72 .-\mathrm{h}$
\end{abstract}

\section{INTRODUCTION}

The microscopic nature of high- $T_{c}$ superconductivity in the cuprates is still under heavy debate after more than two decades of intensive studies. It has been widely accepted that the cuprates may be properly considered as doped Mott insulators and the superconductivity arises after the antiferromagnetic (AFM) long range order is destroyed by doping. The challenge is that a typical doped Mott insulator is a strongly correlated electron system in which two electrons staying at the same lattice site will cost a huge energy as compared to other energy scales like the hopping energy. With the failure of conventional perturbative many-body methods, to tackle a doped Mott insulator one usually resorts to either the so-called Gutzwiller projected trial wavefunction or constructing an effective low-energy theory ${ }^{1}$. In the latter approach, the electron fractionalization ${ }^{1}$ [5 is often utilized in order to implement some key features of a doped Mott insulator. Indeed, the fractionalization due to the Mottness is most transparent at halffilling where only the spin degrees of freedom of the electrons are left, with the charge part being totally frozen out. Only by doping can charge carriers emerge which account for a small portion of the total number of the electrons at low doping.

The real challenge is that there exist many different ways of introducing fractionalized elementary objects to implement the same local Mottness, i.e., the no double occupancy constraint. These elementary particles are generally further coupled to some emergent gauge field(s) due to the uncertainty in the fractionalization ${ }^{1}$. In the lack of precise energetic comparison, a full self-consistent development of a potential fractionalization/gauge theory becomes essential in order for it to be convincingly verified or falsified by experiment as well as by general theoretical considerations. In principle, an inappropriate fractionalization would always lead to strong gauge fluctuations rendering the effective theory intractable. Therefore, a stable, well-controlled gauge theory description will be a highly desired candidate for the correct low-energy theory of cuprate superconductors.

We will consider a particular electron fractionalization description with a mutual Chern-Simons gauge structure ${ }^{6}$. Specifically, an electron in this theory is "fractionalized" into an $S=1 / 2$ bosonic spinon and a charge $+e$ bosonic holon, which are minimally coupled to U(1) gauge fields $A^{h}$ and $A^{s}$, which do not have their own dynamics, but are "entangled" by a mutual Chern-Simons topological term

$$
\mathcal{L}_{\mathrm{MCS}}=\frac{i}{\pi} \epsilon^{\mu \nu \lambda} A_{\mu}^{s} \partial_{\nu} A_{\lambda}^{h} .
$$

which is $(2+1)$ D realization of the topological BF theory ${ }^{9}$ Physically it simply means that the spinons and holons will perceive each other as quantized $\pi$ fluxoids 10 . In the limit of zero hole concentration, the spinon condensation can naturally lead to an AFM long-range ordered state. At finite doping, the mutual Chern-Simons term in Eq. (1) captures $\frac{618}{}$ a precise "statistical sign structure" identified $\frac{10 \mid 11}{1 n}$ in the $t-J$ model. By the $\pi$ fluxoids carried by the mobile holons, the AFM state will be destroyed and eventually a superconducting will set in with the holon condensation, and a zero-temperature phase diagram as a function of doping concentration has been recently discussed in Refs. 617. Finally, there are other quantum systems $\frac{12}{15}$ which have the mutual Chern-Simons gauge structures in different contexts.

* Electronic address: yep07@mails.tsinghua.edu.cn 
In this paper, we focus on the superconducting state to develop an effective description based on the mutual ChernSimons gauge theory in Sec. II. Instead of a conventional Ginzburg-Landau theory in description of the fluctuations of the pairing order parameter, we obtain a gauge description of the low-lying collective fluctuations of charge and spin currents, which are essentially the phase fluctuations of the superconducting order parameter. We show that the Meissner effect in such a superconductor is basically controlled by a spin gap. In Sec. III, we further identify a nonBCS-type order parameter $\mathcal{I}$ that characterizes the SC phase coherence. Here $\mathcal{I}$ is related to the correlation function of two Wilson loops ( of $A^{s}$ and $A^{h}$ ) and is topological as it merely depends on the Gaussian linking number under a continuous deformation of the loops. Such a relation is similar to the connection between the Chern-Simons theory and Knot theory first studied in Ref. 16. But by coupling to the matter fields, we show that the spin correlations strongly renormalize $\mathcal{I}$ such that the phase coherence terminates at a critical point where the spin gap vanishes. In Sec. III C, we shall propose a direct experimental probe of such a topological nature of the gauge interaction between the spin and charge degrees of freedom. Finally, in Sec. III D, we show that a fermionic excitation can emerge in the superconducting phase, which is gauge neutral and thus coherent, resembling a Bogoliubov quasiparticle.

\section{EFFECTIVE FIELD THEORY DESCRIPTION OF SUPERCONDUCTIVITY}

\section{A. Lattice mutual Chern-Simons theory}

The lattice field theory formulation of the phase string theory ${ }^{5}$ for a doped Mott insulator has been recently constructed in Refs. 677 in terms of partition function:

$$
\mathcal{Z}=\sum_{\left\{\mathscr{N}^{s}, \mathscr{N}^{h}\right\}} \int D\left[A^{s}, A^{h}\right] D\left[h^{\dagger}, h, b^{\dagger}, b\right] e^{-S}
$$

in which the action $S=\sum_{x} \mathcal{L}$ ( $x$ denotes spacetime coordinates) with

$$
\mathcal{L}=\mathcal{L}_{h}+\mathcal{L}_{s}+\mathcal{L}_{\mathrm{MCS}}
$$

Here the first two terms, $\mathcal{L}_{h}$ and $\mathcal{L}_{s}$, are the Lagrangian densities for the matter fields, holon $h_{i}$ and spinon $b_{i \sigma}$, which carry charge and spin degrees of freedom, respectively. They are given by

$$
\begin{aligned}
& \mathcal{L}_{h}=h_{i}^{\dagger}\left(d_{0}-i A_{0}^{s}-i A_{0}^{e}+\lambda^{h}\right) h_{i}-t_{h} \sum_{\alpha}\left(e^{i A_{\alpha}^{s}+i A_{\alpha}^{e}} h_{i}^{\dagger} h_{i-\hat{\alpha}}+\text { h.c. }\right)+\frac{u_{1}}{2}\left(h_{i}^{\dagger} h_{i}\right)^{2}, \\
& \mathcal{L}_{s}=\sum_{\sigma} b_{i \sigma}^{\dagger}\left(d_{0}-i \sigma A_{0}^{h}+\lambda^{s}\right) b_{i \sigma}-J_{s} \sum_{\alpha, \sigma}\left(e^{i \sigma A_{\alpha}^{h}} b_{i+\hat{\alpha} \sigma}^{\dagger} b_{i-\sigma}^{\dagger}+\text { h.c. }\right)+\frac{u_{2}}{2}\left(\sum_{\sigma} b_{i \sigma}^{\dagger} b_{i \sigma}\right)^{2} .
\end{aligned}
$$

Equations (4) and (5) show that the bosonic holon and spinon fields minimally couple to two lattice U(1) gauge fields, $A_{\mu}^{s}$ and $A_{\mu}^{h}$, respectively, in $2+1$ dimensions $(\mu=\alpha, 0$, with $\alpha=x, y)$. $\left(A_{\mu}^{e}\right.$ is the external electromagnetic field which solely couples to the holons ${ }^{5}$.) The parameters $t_{h}$ and $J_{s}$ are effective hopping integral and superexchange coupling, respectively, and $d_{0}$ is the difference operator defined along the imaginary time axis. The Lagrangian multipliers, $\lambda^{h, s}$, control the total numbers of holons and spinons, and the last terms in $\mathcal{L}_{h, s}$ describe the on-site repulsions, which soften the hard-core boson condition. It is noted that this effective theory is underpinned by a so-called bosonic RVB order parameter: $\Delta^{s} \equiv \sum_{\sigma}\left\langle e^{i \sigma A_{\alpha}^{h}} b_{i+\hat{\alpha} \sigma}^{\dagger} b_{i-\sigma}^{\dagger}\right\rangle \neq 0$, which describes a short-ranged spin liquid state in the spinion background in the underdoped regime of a doped Mott insulator ${ }^{5}$.

The gauge fields, $A_{\mu}^{h}$ and $A_{\mu}^{s}$, capture the essential physics of the phase string effect 5 in a doped Mott insulator via the following lattice mutual Chern-Simons term in Eq. (3)

$$
\mathcal{L}_{\mathrm{MCS}}=\frac{i}{\pi} \epsilon^{\mu \nu \lambda}\left(A_{\mu}^{s}-2 \pi \mathscr{N}_{\mu}^{s}\right) d_{\nu}\left(A_{\lambda}^{h}-2 \pi \mathscr{N}_{\lambda}^{h}\right),
$$

with $\left\{\mathscr{N}^{s, h}\right\}$ the integer fields due to the compactness of the lattice field theory $\sqrt{67}$, which are important in restoring the spin $\mathrm{SU}(2)$ symmetry. Without $\left\{\mathscr{N}^{s, h}\right\}$, Eq. $\sqrt{1}$ is recovered the present theory reduces to the original mutual Chern-Simons gauge theory formulation of the phase string theory in Ref. 8 . 


\section{B. Low-energy effective theory for superconducting phase}

Superconductivity can naturally arise in a saddle-point state of the above mutual Chern-Simons gauge theory, in which holons become Bose-condensed and spinons are gapped $\underline{6}[\underline{6}$. In the following, we shall derive a general effective theory governing the low-energy fluctuations of the gauge fields $A^{h, s}$ around this saddle-point. This new effective theory will replace the conventional Ginzburg-Landau theory to describe the generalized rigidity of a non-BCS superconducting state.

By minimizing the action $S$ in Eq. 22 with regard to $A_{0}^{s}$ and $A_{0}^{h}$, one can find the saddle-point solution of $A^{h, s}$, i.e., $\bar{A}^{h, s}$ defined by

$$
\epsilon^{\alpha \beta} d_{\alpha} \bar{A}_{\beta}^{h}=\pi \delta, \quad \bar{A}_{\alpha}^{s}=0
$$

in the presence of a uniform distribution of the holon condensate with $\delta$ as the holon concentration. One can always choose the temporal components $\bar{A}_{0}^{s, h}=0$ by shifting $\lambda^{h, s}$ in Eqs. 4 and $(5)$. At the saddle point, one has $\overline{\mathscr{N}}^{s, h}=0$. Equation (7) can be understood as that a fictitious uniform flux $\pi \delta$ is generated by the average distribution of the holon condensate via $\bar{A}^{h}$, while the vanishing net flux in $\bar{A}^{s}$ is due to the fact that the spinons are short-range paired with $\left\langle\sum_{\sigma} \sigma b_{i \sigma}^{\dagger} b_{i \sigma}\right\rangle=0$ (where $\langle\ldots\rangle$ denotes the expectation value at the saddle-point).

Around the saddle-point, the gauge field $A_{\mu}^{h}$ may be rewritten as

$$
A_{\mu}^{h}=\bar{A}_{\mu}^{h}+a_{\mu}^{h}
$$

Then an effective low-energy Lagrangian of the "weak" fluctuations of $A_{\mu}^{s}$ and $A_{\mu}^{h}$ around the saddle-point can be derived as follows

$$
\mathcal{L}_{\text {eff }}\left[A^{s}, a^{h}, A^{e}\right]=\frac{g_{\mu}^{h}}{2 \pi^{2}}\left(A_{\mu}^{s}+A_{\mu}^{e}\right)^{2}+\frac{g_{\mu}^{s}}{2 \pi^{2}}\left(\epsilon^{\mu \nu \lambda} \partial_{\nu} a_{\lambda}^{h}\right)^{2}+\frac{i}{\pi} \epsilon^{\mu \nu \lambda} A_{\mu}^{s} \partial_{\nu} a_{\lambda}^{h}
$$

Here the first term on the right-hand-side (rhs) of Eq. (9) arises from $\mathcal{L}_{h}$ in Eq. (4) after considering the holon condensation ${ }^{7}$, with $g_{0}^{h}=\pi^{2} / u_{1}$ and $g_{1}^{h}=g_{2}^{h}=2 \pi^{2} \bar{\rho}_{h} t_{h}$. Note that the gauge field $A^{s}$ is fixed in the unitary gauge by absorbing Goldstone boson field $\partial_{\mu} \theta$ in the holon superfluid $h=\sqrt{\rho_{h}} e^{i \theta}$, and the superfluid density fluctuation $\delta \rho_{h}=\rho_{h}-\bar{\rho}_{h}$ has been integrated out around $\bar{\rho}_{h}=\delta$.

The second term on the rhs of Eqs. (9) originates from the spinon term $\mathcal{L}_{s}$ in Eq. (5) after integrating out the spinon field $b$, with $g_{0}^{s}=\frac{\gamma}{m_{s}}, g_{1}^{s}=g_{2}^{s}=\frac{\kappa}{m_{s}}$ (the details are outlined in Appendix A). Here, $m_{s}$ denotes the spin mass gap which is an important parameter as discussed in Appendix A below Eq. A1). The parameters $\gamma>0$ and $\kappa>0$ as given in Eq. A6. Note that in Refs. 67. by assuming that the state is deep inside the superconducting phase, the limit of $m_{s} \rightarrow \infty$ has been taken such that $g_{\mu}^{s}=0$ there. Equation 9 corresponds to the general case for a finite spin mass gap $m_{s}$.

The last term on the rhs of Eq. $\sqrt{9}$ is the mutual Chern-Simons term with the high-energy fluctuations of $\mathscr{N}^{s, h}$ omitted. Note that the role played by $\mathscr{N}^{s}$ in constructing a topological object like the magnetic flux quantization has been discussed in Ref. 7 and the references therein.

\section{Meissner effect}

To see that the effective Lagrangian $\mathcal{L}_{\text {eff }}$ in Eq. (9) indeed describes a superconducting state, one may further integrate out $A^{s}$ and $a^{h}$ to arrive at a low-energy action

$$
S_{\text {eff }}\left[A^{e}\right]=\int d^{3} x \frac{M_{p}^{2}}{2}\left(\mathbf{A}^{e \perp}\right)^{2}
$$

where the summation $\sum_{x}$ is replaced by integration in the continuum limit, with the external magnetic field $\mathbf{A}^{e \perp}$ fixed in the Coulomb gauge $\left(\nabla \cdot \mathbf{A}^{e \perp}=0\right)$. Here the transverse photon mass $M_{p}$ is given by

$$
M_{p}=\frac{1}{\lambda_{p}}
$$

with the London penetration depth

$$
\lambda_{p}=\sqrt{\lambda_{0}^{2}+\kappa m_{s}^{-1}}
$$


in which $\lambda_{0} \equiv \frac{1}{\sqrt{2 t_{h} \bar{\rho}_{h}}}$.

Therefore, so long as $m_{s} \neq 0$, the system does exhibit the Meissner effect with a finite $\lambda_{p}$ in response to an external magnetic field. The physical electric current $\mathbf{J}^{e}$ satisfies the London equation:

$$
\mathbf{J}^{e} \equiv \frac{\delta S_{\mathrm{eff}}\left[A^{e}\right]}{\delta \mathbf{A}^{e \perp}}=\frac{1}{\lambda_{p}^{2}} \mathbf{A}^{e \perp} .
$$

Equations (11) and (12) will recover the previous result 617 obtained in the "ideal superconductor" limit of $m_{s} \rightarrow \infty$, where the London penetration depth $\lambda_{p}$ approaches the "shortest" $\lambda_{0}$. Namely, in the large spin-gap limit, the superfluidity of a holon condensate is well protected.

However, in the opposite limit of $m_{s} \rightarrow 0$, i.e., with the spin gap closing up, one finds $\lambda_{p} \rightarrow \infty$ in Eq. 12 such that the Meissner effect disappears. It implies that the superconducting phase coherence can be destroyed by the gapless spin excitations at zero temperature, even in the presence of a bare holon condensate with a finite $\lambda_{0}$. This is a peculiar manifestation that the neutral spin degrees of freedom can strongly influence the superconducting condensate. The quantum phase transition of superconductivity occurring here is quit different from the conventional transition of BCS-type as in the latter a charge (superconducting) gap must be closed up at the Fermi surface. Physically, with $m_{s} \rightarrow 0$, the proliferation of neutral spinons will disorder the superconducting phase coherence via the $\pi$-vortices bound to them in the mutual Chern-Simons gauge theory. Such a superconducting phase transition can also occur at a finite temperature at $T=T_{c} \sim m_{s}$ which is of non-BCS-type (see Ref. 17).

Finally it is noted that $m_{s}=0$ with a spinon condensation is a necessary, but not a sufficient condition for the emergence of a true AFM ordering in the mutual Chern-Simons gauge theory. A so-called Bose insulating phase (BI) with $m_{s}=0$ but $\lambda_{0} \neq 0$ has been proposed ${ }^{\sqrt[6]{67}}$ to appear as an intermediate phase between the superconducting and AFM phases. In other words, the present superconducting phase can be terminated at a critical point defined by $m_{s} \rightarrow 0$ before a true AFM order can set in with further reducing doping concentration near the half-filling.

\section{TOPOLOGICAL CHARACTERIZATION OF SUPERCONDUCTIVITY}

The effective low-energy Lagrangian $\mathcal{L}_{\text {eff }}$ in Eq. (9) has replaced the conventional Ginzburg-Landau equation to describe superconductivity in the mutual Chern-Simons theory. Now we examine the following question: What is the essential physical quantity that captures the nature of superconductivity, since the BCS order parameter (as governed by the conventional Ginzburg-Landau equation) no longer explicitly appears in the low-energy effective theory?

To be sure, the off diagonal long order (ODLRO) of superconductivity is still described by the Cooper pairing order parameter in the phase string theory of doped Mott insulators ${ }^{5}$. Nevertheless, since the Cooper pairing amplitude can persist well beyond the superconducting phase, as determined ${ }^{5}$ by the bosonic RVB parameter $\Delta^{s} \neq 0$ and the holon condensation $\langle h\rangle \neq 0$, the low-lying excitations and the phase transition of superconducting phase are essentially characterized by the topological phase fluctuations via the mutual Chern-Simons gauge fields, $A^{s}$ and $a^{h}$, in the low-energy effective Lagrangian (9).

Physically, $A^{s}$ and $A^{h}$ are related to the spin and hole currents in the mutual Chern-Simon gauge theory by the following equations of motion $\frac{788}{78}$

$$
j_{\alpha}^{s}=\frac{1}{\pi} \epsilon^{0 \alpha \beta} E_{\beta}^{s}, \quad j_{\alpha}^{h}=\frac{1}{\pi} \epsilon^{0 \alpha \beta} E_{\beta}^{h},
$$

where the internal "electric" fields in the imaginary time representation $E_{\alpha}^{s, h} \equiv-i \epsilon^{\alpha \mu \nu} d_{\mu} A_{\nu}^{s, h}$ and the spin and charge currents are defined as $j^{s / h} \equiv \frac{\delta \mathcal{L}_{s / h}}{\delta A^{h / s}}$. Hence the low-lying fluctuations of $A^{s}$ and $a^{h}$ in Eq. (9) actually describe the current fluctuations of the spin and charge degrees of freedom, while the amplitude fluctuations of the matter fields remain gapped in the superconducting phase.

\section{A. Wilson loops}

In order to characterize the gauge fluctuations of $A_{\mu}^{s, h}$, a pair of Wilson loops have been introduced $\frac{617}{6}$ :

$$
W^{s, h}[\mathcal{C}] \equiv\left\langle\hat{W}^{s, h}[\mathcal{C}]\right\rangle,
$$

where

$$
\langle\ldots\rangle \equiv \mathcal{Z}^{-1} \int D\left[A^{s}, A^{h}\right] e^{-\sum_{x} \mathcal{L}_{e f f}} \ldots
$$


and

$$
\hat{W}^{s, h}[\mathcal{C}] \equiv \exp \left[i \sum_{x} A_{\mu}^{s, h}(x) J_{\mathcal{C}}^{\mu}(x)\right]
$$

in which the (2+1)-dimensional unit current, for a test holon or a test spinon, $J_{\mathcal{C}}^{\mu}(x)=+1(-1)$ for the link $x \rightarrow x+\hat{\mu}$ $(x+\hat{\mu} \rightarrow x)$, and is zero otherwise on a close loop $\mathcal{C}$.

Physically, the Wilson loop $W^{s}[\mathcal{C}]$ or $W^{h}[\mathcal{C}]$ probes the interaction of a pair of test holons or spinons at a distance $R$, defined by $V^{h}(R)$ or $V^{s}(R)$, via ${ }^{18}$

$$
V^{h, s}(R) \equiv-\lim _{T \rightarrow \infty} \frac{1}{T} \ln W^{s, h}[\mathcal{C}]
$$

if $\mathcal{C}$ is taken as a spacetime rectangle with length $T(R)$ in the imaginary time (spatial) direction. In Refs. 617, in the limit of large spin gap $\left(m_{s} \rightarrow \infty\right)$, one finds $V^{h}(R) \sim \frac{\lambda_{0}^{2}}{2}$ and $V^{s}(R) \sim \frac{\pi}{2 \lambda_{0}^{2}} \ln R$. Namely the holons are deconfined while the spinons are logarithmically confined. It is consistent with the present saddle-point state of holon condensation discussed in Sec. II.

In the presence of a finite spin gap $m_{s}$, we find that the above conclusion remains true, with the the confining potential, between a test spinon and test anti-spinon, modified to

$$
V^{s}(R)=\frac{\pi}{2 \lambda_{p}^{2}} \ln \left(\frac{R}{R^{*}}\right)
$$

where $\lambda_{0}$ is replaced by $\lambda_{p}$ defined in Eq. $(12)$ ( $R^{*}$ is an ultraviolet cutoff of the distance). The result indicates that the spinons will experience a confinement-deconfinement transition at $\lambda_{p} \rightarrow \infty$ or $m_{s} \rightarrow 0$, coinciding with the superconducting phase transition critical point at $T=0$. On the other hand, $V^{h}(R)$ for holons remains deconfined.

\section{B. Topological characterization}

The Wilson loops, $W^{s, h}[\mathcal{C}]$, can provide important information on the confinement/deconfinement of spinons and holons in the superconducting phase as discussed above. However, to understand why $W^{s, h}[\mathcal{C}]$ behave as such, the topological nature of the mutual Chern-Simons effective theory in Eq. 9) has to be further revealed.

For this purpose we define

$$
e^{i \mathcal{I}} \equiv \mathcal{K}^{-1}\left\langle\hat{W}^{s}\left[\mathcal{C}_{1}\right] \hat{W}^{h}\left[\mathcal{C}_{2}\right]\right\rangle
$$

with the normalization factor

$$
\mathcal{K} \equiv W^{h}\left[\mathcal{C}_{1}\right] W^{s}\left[\mathcal{C}_{2}\right] .
$$

Let us first consider the case of a pure mutual Chern-Simons term (6) without coupling to the matter fields [i.e., omitting the first two terms in Eq. [9]], a straightforward calculation shows

$$
\mathcal{I}=\pi \Theta
$$

where $\Theta=0, \pm 1, \pm 2, \cdots$, denotes the Gaussian linking number, which is the winding number of the close loops, $\mathcal{C}_{1}$ and $\mathcal{C}_{2}$, as shown in Fig. 1 (a) and (b). To obtain Eq. 21), we write down the explicit expression of $\mathcal{I}$ in a pure mutual Chern-Simons gauge theory,

$$
e^{i \mathcal{I}}=\mathcal{K}^{-1} \int D\left[A^{s}, a^{h}\right] e^{-\int d^{3} x\left(\frac{i}{\pi} \epsilon^{\mu \nu \lambda} A_{\mu}^{s} \partial_{\nu} a_{\lambda}^{h}-A_{\mu}^{s} J_{\mu}^{\mathcal{C}_{1}}-a_{\mu}^{h} J_{\mu}^{\mathcal{C}_{2}}\right)}
$$

where, the unit spacetime currents $J^{\mathcal{C}_{1}}$ and $J^{\mathcal{C}_{2}}$ keep track of spacetime loops, $\mathcal{C}_{1}$ and $\mathcal{C}_{2}$, respectively. Integrating over $A^{s}$ leads to a flux constraint on $a^{h}$ configuration, namely, $\epsilon^{\mu \nu \lambda} \partial_{\nu} a_{\lambda}^{h}=\pi J_{\mu}^{C_{1}}$. It means that the gauge field $a^{h}$ forms a spacetime closed $\pi$-fluxtube along $\mathcal{C}_{1} \cdot \int d^{3} x a_{\mu}^{h} J_{\mu}^{\mathcal{C}_{2}}=\oint_{\mathcal{C}_{2}} a_{\mu}^{h} d x_{\mu}$ denotes the spacetime flux piercing $\mathcal{C}_{2}$. Noting that there are $\Theta$ times for the fluxtube $\mathcal{C}_{1}$ piercing $\mathcal{C}_{2}$, one arrives at $e^{i \mathcal{I}}=e^{i \pi \Theta}$, with $\mathcal{K}=1$. Ignoring the $2 \pi$-periodicity, we thus obtain Eq. 21]. In Witten's seminal paper $\frac{\sqrt{16}}{}$, a similar Gaussian linking number structure was found in the Chern-Simons gauge theory, which is related to the Jones polynomial in the Knot theory. 


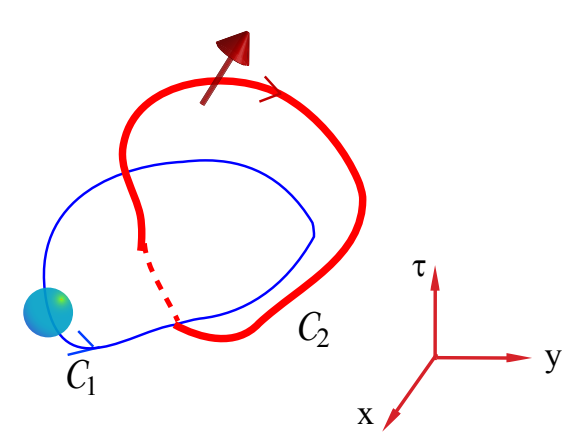

(a) $\Theta=1$

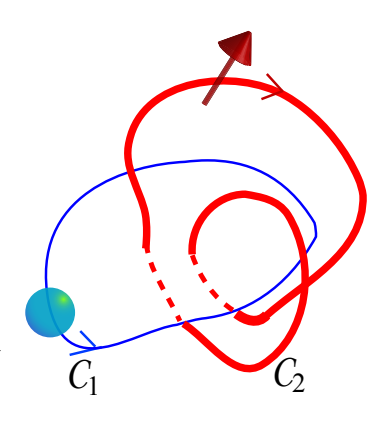

(b) $\Theta=2$

FIG. 1: (Color online). Schematic picture of two loops $\mathcal{C}_{1}$ and $\mathcal{C}_{2}$ in $(2+1)$-dimensional Euclidean spacetime. $\mathcal{C}_{1}$ is an arbitrary loop but restricted inside spatial plane (i.e., the xy plane) at some imaginary time. $\mathcal{C}_{2}$ is an arbitrary loop with a definite Gaussian linking number $\Theta \in \mathbb{Z}\left(\Theta=1\right.$ in (a); $\Theta=2$ in (b)) that characterizes the number of times that $\mathcal{C}_{2}$ winds around $\mathcal{C}_{1}$. The ball (in blue) and the arrow (in red) stand for the test holon and test spinon, respectively. The arrows on the two loops indicate the direction of test spinon current and test holon current.

In Fig. 2, a gedankenexperiment is shown to illustrate the case of winding number $\Theta=1$ in the $(2+1)$-dimensional Euclidean spacetime, with a pair of test spinon and antispinon creating and annihilating on a close loop $\mathcal{C}_{2}$. If the background spin degrees of freedom are gapped with $m_{s} \longrightarrow \infty$, the test spinons cannot induce spin excitations from the ground state and thus the net contribution to $e^{i \mathcal{I}}$ comes from the $\pi$ flux phase shift $($ with $\Theta=1)$ mentioned above due to the pure mutual Chern-Simons action.

On the other hand, if the spin gap $m_{s}$ becomes finite, then the spin excitations can be induced from the spinon background to renomalize $\mathcal{I}$. Now we consider the full $\mathcal{L}_{\text {eff }}$ given in Eq. (9). By choosing the close loop $\mathcal{C}_{1}$ of the test holon within the spatial $x y$-plane, one finally arrives at the following compact result (for the details, see Appendix $\mathrm{B})$ :

$$
\mathcal{I}=\pi \Theta\left(\lambda_{0} / \lambda_{p}\right)^{2}
$$

We see that $\mathcal{I}$ remains to be real and positive as a topological quantity, which is invariant under a continuous deformation of loops $\mathcal{C}_{1}$ and $\mathcal{C}_{2}$ for a given Gaussian linking number $\Theta$, as illustrated in Fig. 1 . But $\mathcal{I}$ is renormalized by a factor $\left(\lambda_{0} / \lambda_{p}\right)^{2}$ which is solely determined by the spin gap $m_{s}$. In particular, in the limit of $m_{s} \rightarrow 0$, one finds $\mathcal{I} \rightarrow 0$.

If one rewrites Eq. 22 by

$$
\lambda_{p}=\lambda_{0} \cdot \sqrt{\pi / \mathcal{I}}
$$

where $\lambda_{p}$ becomes a function of topological order parameter $\mathcal{I}$ for a given Gaussian linking number $\Theta=1$, then the limit $\lambda_{p} \rightarrow \infty$, i.e., the disappearance of superconductivity, will correspond to $\mathcal{I}=0$. In other words, the topological quantity $\mathcal{I}$ can be regarded as the superconducting order parameter in the effective theory of Eq. (9), and the quantum phase transition at $T=0$ occurs when $\mathcal{I}$ gets fully screened by the gapless spin excitations at $m_{s}=0$.

As illustrated in Fig. 1 $\mathcal{I} \neq 0$ indicates that a test spinon can be always "felt" by a vortex-like current loop of a test holon if their mutual winding number $\Theta \neq 0$. Physically, since the holons are in the Bose condensed state, a spinon excitation will then induce a supercurrent vortex response from the condensed holons throughout the sample to form a so-called spinon-vortex 19 . This is the origin of spinon confinement discussed above, since each spinon-vortex composite is logarithmically divergent in energy which will result in vortex-antivortex confinement. In a non-superconducting phase where the spinons become deconfined due to the vortex-antivortex disassociation and proliferation, one finds $\mathcal{I}=0$ in the gedankenexperiment shown in Fig. 2. Namely, $\mathcal{I}=0$ describes a phase disordered superconductor.

\section{Experimental prediction}

We propose an experimental setup to probe the topological characterization $\mathcal{I}$ of the superconducting phase in Fig. 3. Note that $\mathcal{I} \neq 0$ reflects the mutual statistical interaction between spin and charge degrees of freedom (cf. Fig. 1 . 

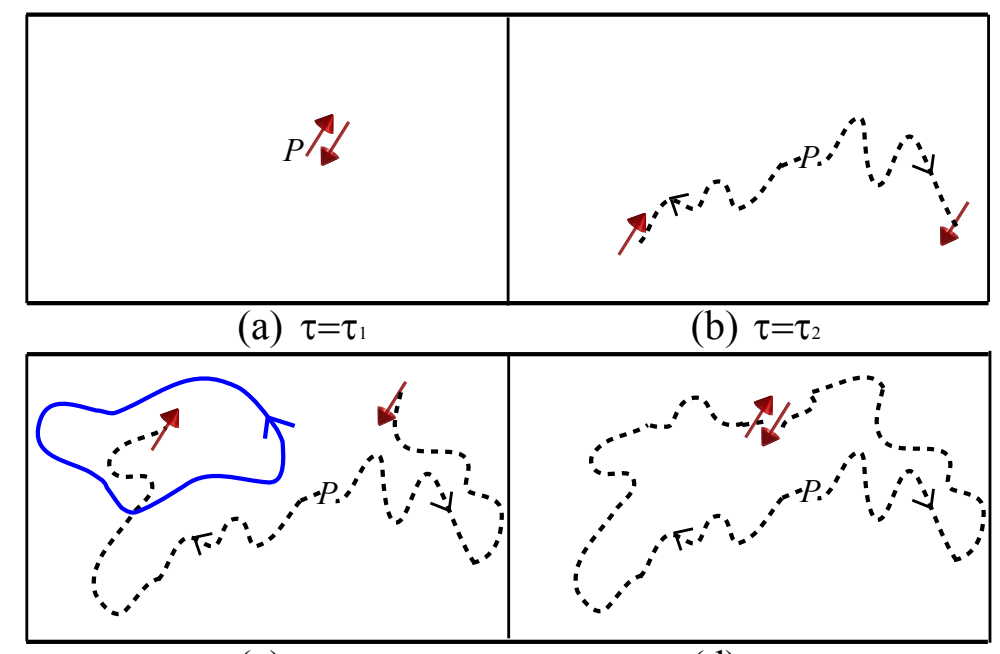

(c) $\tau=\tau_{3}$

(d) $\tau=\tau_{4}$

FIG. 2: (Color online). Gedankenexperiment (Gaussian linking number $g=1$ for simplicity). (a-d) are four snapshots of spatial plane subsequently at imaginary time $\tau_{1}<\tau_{2}<\tau_{3}<\tau_{4}$. (a) A pair of test spinons with opposite spin indices are created at a spatial site "P" at $\tau_{1}$. (b) Two spinons arrive at new spatial positions at $\tau_{2}$ via the dashed trajectories. (c) At $\tau_{3}$, a holon current circuit $\mathcal{C}_{1}$ is located in plane, indicating that an Aharonov-Bohm phase around $\mathcal{C}_{1}$ is picked up by the test holon. (d) At $\tau_{4}$, the pair of test spinons meet each other again at some position and then are annihilated into vacuum simultaneously.

By using the idea illustrated in the gedankenexperiment of Fig. 2, one may inject free "test" spinons by heating up the middle semi-island part of the sample in Fig. 3 to a temperature slightly higher than $T_{c}$, such that free spinons can be excited and flow towards the lower $T$ end connecting to the outside superconducting ring. With $\mathcal{I} \neq 0$ inside the ring, one expects that such an injection of free spinons can generate supercurrents in the superconducting ring in a form of significant noise spikes due to the unpolarized spinon-vortex effect.

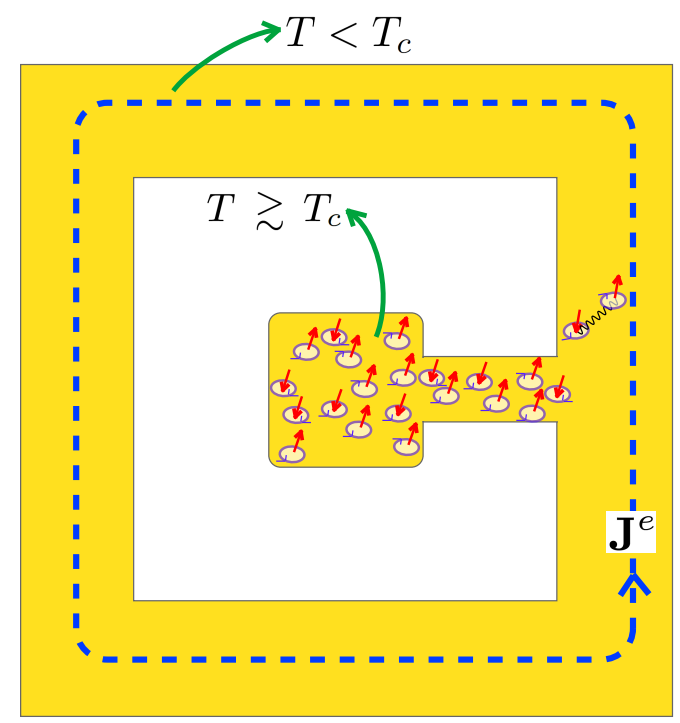

FIG. 3: (Color online) A proposed experiment to detect mutual statistics in a mutual Chern-Simons superconductor. The temperature in the middle semi-island is raised slightly above the superconducting critical temperature $T_{c}$, while the outside part remains below $T_{c}$ and spinons are confined into vortex-antivortex pairs. The confinement is indicated by the black wavy line between two spinons. The small directional discs (red arrows) represent vortices (spinons). In such a mutual Chern-Simons

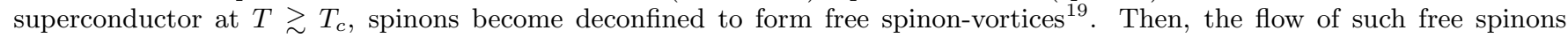
from the semi-island towards the cooler end connecting to the outside superconducting ring is expected to generate random supercurrents in the latter as indicated by $\mathbf{J}^{e}$ (blue dashed line).

Instead of raising the temperature, one may also apply an in-plance magnetic field $B^{e}$ to reduce the spinon mass 
gap $m_{s}$ inside the semi-island part by $\widetilde{m_{s}}=m_{s}-\frac{1}{2} g_{L} \mu_{B} B^{e}$, where $g_{L}$ is the Landé factor and $\mu_{B}$ is the Bohr magneton. Then a sufficiently strong $B^{e}$ may drive the semi-island part into a spinon-vortex liquid state with $\mathcal{I}=0$ via the Zeeman effect 19 . At the same time, the gradient of $B^{e}$ can also naturally cause the free spinons flow into the superconducting ring to induce the similar supercurrent response shown in Fig. 3

\section{Emergence of fermionic excitations: A proof-of-principle derivation}

The mutual Chern-Simons theory given in Eq. (3) is an all-boson description of doped Mott insulators. In the above subsections we have studied the low-lying collective fluctuations of this theory based on the effective Lagrangian (9). An important question that arises naturally is if and how a low-energy fermionic excitation can emerge in such an all-boson theory, which is independent of the above collective excitations.

To give a proof of principle, we first note that the mutual Chern-Simons term in Eq. (9) can be replaced by an auxiliary Lagrangian in real-time representation:

$$
\mathcal{L}_{\text {aux }}=\sum_{f=1,2}\left(\bar{\Psi}_{f} i \not \supset \Psi_{f}-\Delta \bar{\Psi}_{f} \Psi_{f}\right)
$$

where $\Psi_{f}$ is an auxiliary 4-component Dirac spinor in (2+1)-dimensional spacetime with "flavor" indices $f=1,2$. $\not D D \equiv \gamma^{\mu} \mathcal{D}_{\mu}$. Here $\gamma$-matrices are defined in 4-dimensional reducible representation of 2-dimensional Clifford algebra, i.e., $\gamma^{0}=\operatorname{diag}\left(\sigma^{3},-\sigma^{3}\right), \gamma^{\alpha}=\operatorname{diag}\left(\sigma^{\alpha},-\sigma^{\alpha}\right)$, where $\alpha=1,2$ and $\sigma^{1,2,3}$ are Pauli matrices. The covariant derivative $\mathcal{D}_{\mu}$ is defined as $\mathcal{D}_{\mu} \equiv \partial_{\mu}+i A_{\mu}^{s}+i \tau_{3} a_{\mu}^{h}$ The $4 \times 4$ matrix $\tau_{3}=\operatorname{diag}(\mathbb{I},-\mathbb{I})$, where $\mathbb{I}$ is the $2 \times 2$ unit matrix. The second term in Eq. (24) is mass term with $\Delta>0$.

$\mathcal{L}_{\text {aux }}$ preserves a $U(1)_{A} \times U(1)_{V}$ gauge symmetry where subscripts $A$ and $V$ denote axial vector type and ordinary vector type $U(1)$ gauge invariance, respectively. It originates from the fact that $A_{\mu}^{s}$ is an ordinary vector field with gauge group generator " 1 " while $a^{h}$ is an axial vector field with gauge group generator $\tau_{3} 8$. The parity and timereversal symmetries are conserved even in the presence of finite mass gap which may be expressed as: $\Delta \bar{\Psi}_{f} \Psi_{f}=$ $\Delta \Psi_{f}^{\dagger} \gamma_{0} \Psi_{f}=\Delta \Psi_{f+}^{\dagger} \sigma^{3} \Psi_{f+}-\Delta \Psi_{f-}^{\dagger} \sigma^{3} \Psi_{f-}$, where, $\Psi_{f+}\left(\Psi_{f-}\right)$ is upper (lower) 2-component spinor of $\Psi_{f}$. Here, \pm denotes the chirality 20 . In the case of parity ( $\hat{\mathbb{P}}$ is parity operator),

$$
\Psi_{f} \rightarrow \hat{\mathbb{P}} \Psi_{f}=\left(\begin{array}{cc}
0 & \sigma^{1} \\
\sigma^{1} & 0
\end{array}\right)\left(\begin{array}{l}
\Psi_{f+} \\
\Psi_{f-}
\end{array}\right)=\left(\begin{array}{c}
\sigma^{1} \Psi_{f-} \\
\sigma^{1} \Psi_{f+}
\end{array}\right)
$$

It is easy to check that the mass term is invariant under such parity transformation in (2+1)-dimensional spacetime. Integrating over $\Psi_{f}$ leads to a pure mutual Chern-Simons term by ignoring Maxwell terms ( $\propto$ momentum squared) in the one-loop quantum correction (Fig. (4) in the long wavelength limit $|p| \ll \Delta$. The key steps of derivation are given as follows. As shown in Fig. 44 the vacuum polarization for $A^{s}$ and $a^{h}$ at one-loop leve ${ }^{21 / 22}$ ( $p$ is a 3-dimensional

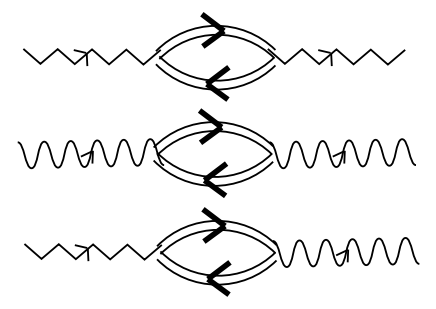

FIG. 4: One-loop quantum correction. Zigzag, wavy, and double lines represent $A^{s}, a^{h}$, and $\Psi$, correspondingly. The first two diagrams correspond to Maxwell terms, while the last one gives the non-vanishing mutual Chern-Simons vertex.

Euclidean momentum):

$$
\Pi(p)=\frac{\mathcal{N}_{f}}{4 \pi p^{2}}\left[2 \Delta+\frac{p^{2}-4 \Delta^{2}}{p} \arcsin \left(\frac{p}{\left(p^{2}+4 \Delta^{2}\right)^{1 / 2}}\right)\right] .
$$

where the total flavor number $\mathcal{N}_{f}=2$. The expansion around small $p$ is given by

$$
\Pi(p)=\frac{\mathcal{N}_{f}}{6 \pi \Delta}-\frac{\mathcal{N}_{f} p^{2}}{60 \pi \Delta^{3}}+\frac{3 \mathcal{N}_{f} p^{4}}{1120 \pi \Delta^{5}}+\cdots .
$$


The propagators of gauge fields can be constructed in the Landau gauge and the Maxwell terms read out: $-\frac{\left(f_{\mu \nu}^{s}\right)^{2}}{12 \pi \Delta}-$ $\frac{\left(f_{\mu \nu}^{h}\right)^{2}}{12 \pi \Delta}$ with $f_{\mu \nu}^{s} \equiv \partial_{\mu} A_{\mu}^{s}-\partial_{\nu} A_{\mu}^{s}$ and $f_{\mu \nu}^{h} \equiv \partial_{\mu} a_{\mu}^{h}-\partial_{\nu} a_{\mu}^{h}$. Meanwhile, the nonvanishing mutual Chern-Simons term for $A^{s}$ and $a^{h}$ in Fig. 4 is also radiatively generated without breaking parity and time-reversal symmetries. Note that, upper(lower)-component spinor has mass $\Delta(-\Delta)$ :

$$
\Pi_{\mu \nu}^{\sigma}(p)=-\sigma \Delta \mathcal{N}_{f} \int \frac{d^{3} k}{(2 \pi)^{3}} \frac{\operatorname{Tr}\left(\widetilde{\gamma}_{\mu} \widetilde{\gamma}_{\lambda} p^{\lambda} \widetilde{\gamma}_{\nu}\right)}{\left(k^{2}+\Delta^{2}\right)\left((p+k)^{2}+\Delta^{2}\right)}=\frac{\sigma \Delta \mathcal{N}_{f}}{4 \pi} \epsilon_{\mu \nu \lambda} p^{\lambda} \int_{0}^{1} d \ell \frac{1}{\sqrt{\Delta^{2}+\ell(1-\ell) p^{2}}}
$$

where, the superscript $\sigma=\uparrow(1), \downarrow(-1)$ denoting upper and lower 2-component spinor contributions respectively, and $\widetilde{\gamma}_{\mu}=i\left\{\sigma^{1}, \sigma^{2}, \sigma^{3}\right\}$ with identity: $\operatorname{Tr}\left(\widetilde{\gamma}_{\mu} \widetilde{\gamma}_{\nu} \widetilde{\gamma}_{\lambda}\right)=2 \epsilon_{\mu \nu \lambda}$. In the sufficiently low momentum transfer limit $(p \rightarrow 0)$, it is expanded as power series of $p$ :

$$
\Pi_{\mu \nu}^{\sigma}(p)=\frac{\sigma \mathcal{N}_{f}}{4 \pi} \epsilon_{\mu \nu \lambda} p^{\lambda}\left(1-\frac{p^{2}}{12 \Delta^{2}}+\frac{p^{4}}{80 \Delta^{4}}+\cdots\right)
$$

In Eq. (26), the sign factor " $\sigma$ " renders vanishing of Abelian Chern-Simons terms $\left(\sim \epsilon^{\mu \nu \lambda} A_{\mu}^{s} \partial_{\nu} A_{\lambda}^{s}\right.$ and $\left.\sim \epsilon^{\mu \nu \lambda} a_{\mu}^{h} \partial_{\nu} a_{\lambda}^{h}\right)$. But due to the structure of matrix $\tau_{3}$, the crossing term, i.e the mutual Chern-Simons term survives as expected. Mathematically, the above quantum corrections are organized as below:

$$
\frac{1}{2} \sum_{\sigma= \pm}\left(A_{\mu}^{s}+\sigma a_{\mu}^{h}\right) \frac{\sigma \mathcal{N}_{f}}{4 \pi} \epsilon^{\mu \nu \lambda} \partial_{\nu}\left(A_{\lambda}^{s}+\sigma a_{\lambda}^{h}\right)=\frac{1}{\pi} \epsilon^{\mu \nu \lambda} A_{\mu}^{s} \partial_{\nu} a_{\lambda}^{h}
$$

where the Maxwell terms are ignored in the long wavelength limit.

It is important to note that the fermionic auxiliary field $\Psi_{f}$ is not gauge-neutral as it still couples to $A_{\mu}^{s}$ and $a_{\mu}^{h}$ in Eq. 24). A true fermion excitation of quasiparticle should be gauge-neutral without knowing $A^{s}$ and $a^{h}$. It is only possible if there exists a residual attractive potential such that a holon and a spinon excited from the condensates can form a bound state properly with the auxiliary field $\Psi_{f}$. The resulting object $\Psi$ will be both fermionic and coherent because all statistical gauge charges carried by $\Psi_{f}$ can be exactly canceled by that of the spinon and holon partners. In Fig. 5, the spacetime loop of a composite-particle $\Psi$ is illustrated as a gauge neutral and thus physically observable elementary excitation at low energies.

In particular, it carries the right quantum number as a Bogoliubov quasiparticle. As noted before, to produce the right prefactor $i / \pi$ of the pure mutual Chern-Simons term, $\Psi_{f}$ has totally $2 \times 2=4$ branches of positive energy solutions, which are consistent with the existence of totally four quasiparticle nodal regions in the Brillouin zone, although the detailed energy spectrum depends on the residual attractive potential which goes beyond the present long-wavelength effective description. On the other hand, the existence of a nodal fermionic Bogoliubov quasiparticle has been recently demonstrated based on a ground state wavefunction approach to the $t-J$ model in the phase string formulation, in which the quasiparticle as a similar bound state has been explicitly shown 23 . Therefore, the present field-theory formulation provides a proof-of-principle for a fermionic quasiparticle mode emerging in the mutual Chern-Simons theory.

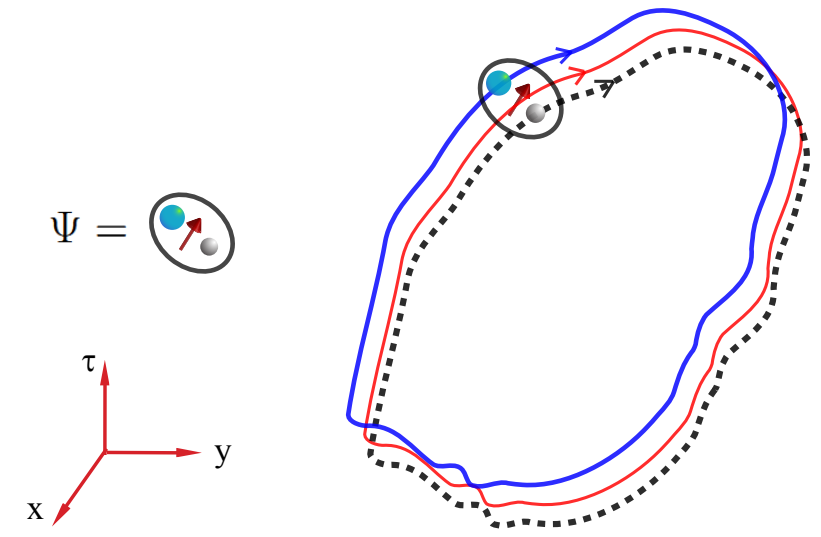

FIG. 5: (Color online). $\Psi$ is composed by a spinon (red arrow), a holon (blue ball) and a Dirac fermion (small grey ball $\Psi_{f}$ ). Its spacetime loop is composed by three loops rendering gauge charge neutrality. 


\section{CONCLUSION}

In this work, we have obtained an effective theory of the low-lying fluctuations in the superconducting state of the mutual Chern-Simons theory. In contrast with a conventional Ginzburg-Landau action for a BCS superconductor, the present effective description in Eq. (9) deals with peculiar phase fluctuations in the doped Mott insulator via a pair of mutual Chern-Simons gauge fields, which are related to the fluctuations of charge and spin currents of the condensate.

The superconducting phase coherence is found to be properly characterized by a topological order parameter which measures the mutual entanglement of the charge and spin currents in the low-energy effective theory. In particular, a zero-temperature quantum phase transition is shown to occur, due to the vanishing of this topological order parameter, when the spin gap is reduced to zero at low doping.

We have discussed a possible experimental probe into the long-range entanglement between the supercurrents and the neutral spin excitations as a unique signature of this non-BCS superconducting state. We have also given a proof-of-principle how a fermionic quasiparticle can emerge in the mutual Chern-Simons theory. Together with the previously identified flux quantization $\left[\frac{618}{1}\right.$ at $h c / 2 e$, the non-BCS superconductivity in the mutual Chern-Simons theory is well established.

\section{Acknowledgments}

We acknowledge stimulating discussions with Z. C. Gu, S.-P. Kou, Z.-X. Liu, C.-S. Tian, Y. Wang, Z. Wang, N. $\mathrm{Xu}$, and Y.-Z. You. This work is supported by NSFC grant No. 10834003, by MOST National Program for Basic Research grant No. 2009CB929402, No. 2010CB923003.

${ }^{1}$ For a review, see, P. A. Lee, N. Nagaosa, and X. G. Wen, Rev. Mod. Phys. 78, 17 (2006).

2 S. A. Kivelson, D. S. Rokhsar and J. P. Sethna. Phys. Rev. B 35, 8865 (1987).

3 Z. Zou and P.W. Anderson, Phys. Rev. B 37, 627 (1988).

4 T. Senthil and Matthew P. A. Fisher, Phys. Rev. B 62, 7850 (2000).

5 Z. Y. Weng, Int. J. Mod. Phys. B 21, 773 (2007).

${ }_{7}^{6}$ P. Ye, C. S. Tian, X. L. Qi, and Z. Y. Weng, Phys. Rev. Lett. 106, 147002 (2011);

7 P. Ye, C. S. Tian, X. L. Qi, and Z. Y. Weng, Nucl. Phys. B 854, 815 (2012).

8 S. P. Kou, X. L. Qi, and Z. Y. Weng, Phys. Rev. B 71, 235102 (2005).

9 D. Birmingham, M. Blau, M. Rakowski, and G. Thompson, Phys. Rep. 209, 129 (1991).

10 Z. Y. Weng, D. N. Sheng, Y.-C. Chen, and C. S. Ting, Phys. Rev. B 55, 3894 (1997).

11 K. Wu, Z. Y. Weng, and J. Zaanen, Phys. Rev. B 77, 155102 (2008).

12 V. M. Galitski, G. Refael, M. P. A. Fisher, and T. Senthil, Phys. Rev. Lett. 95, 077002 (2005).

13 M. C. Diamantini, P. Sodano, and C. A. Trugenberger, Nucl. Phys. B 448, 505 (1991); Nucl. Phys. B 474, 641 (1996).

14 G. Y. Cho and J. E. Moore, Ann. of Phys. 326, 1515 (2011).

15 C. Xu and S. Sachdev, Phys. Rev. B 79, 064405 (2009).

16 E. Witten, Commun. Math. Phys. 121, 351 (1989).

17 J. W. Mei and Z. Y. Weng, Phys. Rev. B 81, 014507 (2010).

18 J. Smit, Introduction to Quantum Fields on a Lattice. (Cambridge, 2003)

19 X. L. Qi and Z. Y. Weng, Phys. Rev. B 76, 104502 (2007).

20 C. Vafa and E. Witten, Commun. Math. Phys. 95, 257 (1984).

${ }^{21}$ Robert D. Pisarski, Phys. Rev. D, 29, 2423 (1984).

22 S. Deser, R. Jackiw, and S. Templeton, Ann. Phys., 140, 372 (1982).

23 Z. Y. Weng, New J. Phys. 13, 103039 (2011).

${ }^{24}$ For a review, see, C. Schubert, Phys. Rep. 355, 73 (2001).

${ }^{25}$ Greiner and Reinhardt, Field Quantization, (Spinger-Verlag, 1996). 


\section{Appendix A: Path-integration over spinons}

First of all, we note that the spinon Lagrangian $\mathcal{L}_{s}$ in Eq. (5) may be further simplified to a $\mathrm{CP}(1)$ form in the long-wavelength, low-energy regime as follows

$$
\mathcal{L}_{s} \simeq \sum_{\sigma} \frac{1}{2 g}\left[\left|\left(\partial_{\mu}-i \sigma A_{\mu}^{h}\right) z_{\sigma}\right|^{2}+m_{s}^{2}\left|z_{\sigma}\right|^{2}\right]
$$

Here the temporal component is rescaled by the spin wave velocity $c_{s} \equiv \sqrt{J_{s}\left(\lambda_{s}+4 J_{s}\right)}$ with the coupling constant $g=c_{s} /\left(2 J_{s}\right)$, and $m_{s}$ is treated as a dynamically generated spinon mass enforcing $\sum_{\sigma}\left|z_{\sigma}\right|^{2}=1$.

The problem of studying the fluctuation of $A_{\mu}^{h}$, or $a_{\mu}^{h}$, in Eq. A1 is then transformed into calculating the vacuum polarization tensor in (2+1)-dimensional scalar quantum electrodynamics in the presence of a uniform background magnetic field of $\bar{A}^{h}$ defined in Eq. (7). The technical challenge arises from the fact that because of $\bar{A}^{h}$, the momentum is no longer a good quantum number. Nevertheless the issue is resolved by using the so-called "string-inspired" formalism 24 . Following the procedures and conventions in Ref. 24, we obtain the polarization tensor:

$$
\Pi_{\mu \nu}(k)=2 \times \frac{-1}{(4 \pi)^{\frac{D}{2}}} \times \int_{0}^{\infty} \frac{d T}{T} T^{2-\frac{D}{2}} e^{-m_{s}^{2} T} \operatorname{det}^{-\frac{1}{2}}\left[\frac{\sin (Z)}{Z}\right] \int_{0}^{1} d \ell I^{\mu \nu}
$$

where the prefactor 2 comes from the summation over the spin index, $T$ denotes the Schwinger proper time, and spacetime dimension $D=3$. Here $Z$ is a $3 \times 3$ matrix, defined by $Z=\pi F T$ with

$$
F=\left(\begin{array}{ccc}
0 & 0 & 0 \\
0 & 0 & \pi \delta \\
0 & -\pi \delta & 0
\end{array}\right)
$$

The determinant in Eq. A2 can be simply factorized into:

$$
\operatorname{det}^{-\frac{1}{2}}\left[\frac{\sin (Z)}{Z}\right]=\frac{\pi \delta T}{\sinh (\pi \delta T)}
$$

and the transversality of Lorentz tensor $I_{\mu \nu}$ is given by

$$
I_{\mu \nu}=-(1-2 \ell) \frac{\sinh (\pi \delta T(1-2 \ell))}{\sinh (\pi \delta T)}\left(k_{\mu} k_{\mu}-\delta_{\mu \nu} k^{2}\right)
$$

By further restoring the spin wave velocity $c_{s}$, we arrive at the "Maxwell" term on the rhs of Eq. (9) which describes the fluctuation $a_{\mu}^{h}$, with the coefficients given by

$$
\gamma=\frac{\vartheta}{12 c_{s}}, \quad \kappa=\frac{\vartheta c_{s} \pi}{12}
$$

in which the dimensionless coefficient $\vartheta$ depends on the dimensionless quantity $\frac{c_{s} \pi \delta}{m_{s}}$ as follows

$$
\vartheta=\frac{\int_{0}^{\infty} \frac{d T}{T^{1 / 2}} e^{-T} \int_{0}^{1} d \ell(1-2 \ell) \cdot \pi c_{s} \delta T m_{s}^{-2} \sinh \left((1-2 \ell) \pi c_{s} \delta T m_{s}^{-2}\right) \cdot\left(\sinh \left(\pi c_{s} \delta T m_{s}^{-2}\right)\right)^{-2}}{\int_{0}^{\infty} \frac{d T}{T^{1 / 2}} e^{-T} \int_{0}^{1} d \ell(1-2 \ell)^{2}}
$$

Note that $\vartheta=1$ in the limit of the absence of "magnetic flux" in $\bar{A}^{h}$.

\section{Appendix B: Derivation details of topological order parameter $\mathcal{I}$}

To obtain $\mathcal{I}$, we first note that the Lagrangian with holon and spinon currents circulating on loops $\mathcal{C}_{1,2}\left(m^{*}=\right.$ $\left.\left(2 t_{h}\right)^{-1}\right)$

$$
L_{\mathrm{SC}}\left[\mathcal{C}_{1}, \mathcal{C}_{2}\right]=\frac{\delta}{2 m^{*}}\left(A_{\alpha}^{s}\right)^{2}+\frac{1}{2 u_{1}}\left(A_{0}^{s}\right)^{2}+\frac{i}{\pi} \epsilon_{\mu \nu \lambda} a_{\mu}^{h} \partial_{\nu} A_{\lambda}^{s}+\frac{1}{2} \frac{g_{\mu}}{\pi^{2}}\left(\epsilon_{\mu \nu \lambda} \partial_{\nu} a_{\lambda}^{h}\right)^{2}-i A_{\mu}^{s} J_{\mu}^{\mathcal{C}_{1}}-i a_{\mu}^{h} J_{\mu}^{\mathcal{C}_{2}}
$$


is quadratic in the gauge fields thus allows rigorously integrating out $A^{s}$ and $a^{h}$ in the generating functional

$$
\left\langle\hat{W}^{s}\left[\mathcal{C}_{1}\right] \hat{W}^{h}\left[\mathcal{C}_{2}\right]\right\rangle=\int D\left[A^{s} a^{h}\right] e^{-\int d^{3} x L_{\mathrm{SC}}\left[\mathcal{C}_{1}, \mathcal{C}_{2}\right]}=\text { Const. } \times \int D\left[a^{h}\right] \exp \left\{-\int d^{3} x\left(\frac{G_{\mu}}{2 \pi^{2}}\left(\epsilon_{\mu \nu \lambda} \partial_{\nu} a_{\lambda}^{h}\right)^{2}-i a_{\mu}^{h} J_{\mu}\right)\right\}
$$

in which we have defined

$$
\begin{gathered}
G_{\mu}=g_{\mu}+\left(u_{1}, \frac{m^{*}}{\delta}, \frac{m^{*}}{\delta}\right)_{\mu}, \quad J_{0}=J_{0}^{\mathcal{C}_{2}}-\frac{i}{\pi} \frac{m^{*}}{\delta}\left(\partial_{1} J_{2}^{\mathcal{C}_{1}}-\partial_{2} J_{1}^{\mathcal{C}_{1}}\right), \\
J_{1}=J_{1}^{\mathcal{C}_{2}}-\frac{i}{\pi}\left(u_{1} \partial_{2} J_{0}^{\mathcal{C}_{1}}-\frac{m^{*}}{\delta} \partial_{0} J_{2}^{\mathcal{C}_{1}}\right), \quad J_{2}=J_{2}^{\mathcal{C}_{2}}-\frac{i}{\pi}\left(\frac{m^{*}}{\delta} \partial_{0} J_{1}^{\mathcal{C}_{1}}-u_{1} \partial_{1} J_{0}^{\mathcal{C}_{1}}\right)
\end{gathered}
$$

and absorbed terms unrelated to mutual correlation between $\mathcal{C}_{1}$ and $\mathcal{C}_{2}$ into Const. which will be canceled by the denominator in the definition of $\mathcal{I}$.

Adopting the "deformed" Lorentz gauge condition,

$$
\frac{1}{G_{0}} \partial_{0} a_{0}^{h}+\frac{1}{G_{1}} \partial_{1} a_{1}^{h}+\frac{1}{G_{2}} \partial_{2} a_{2}^{h}=0
$$

and integrating out $a^{h}$ in the Feynman gauge 25 , we get

$$
\left\langle\hat{W}^{s}\left[\mathcal{C}_{1}\right] \hat{W}^{h}\left[\mathcal{C}_{2}\right]\right\rangle=\text { Const. } \times \exp \left\{-\frac{1}{2} \frac{\pi^{2}}{G_{0} G_{1} G_{2}} \int d^{3} x \int d^{3} y \sum_{\mu} G_{\mu} J_{\mu}(x) J_{\mu}(y) f(x, y)\right\}
$$

in which

$$
f(x, y) \equiv \int \frac{d^{3} k}{(2 \pi)^{3}}\left(\sum_{\lambda} \frac{k_{\lambda}^{2}}{G_{\lambda}}\right)^{-1} e^{-i(x-y) \cdot k}
$$

Expanding the integrand in Eq. B4 and extracting mutual correlation terms between $\mathcal{C}_{1}$ and $\mathcal{C}_{2}$ only, and noting that $\int d^{3} x J_{\mu}^{\mathcal{C}_{i}}(x)=\oint_{\mathcal{C}_{i}} d x_{\mu}, i=1,2$, we get

$$
\begin{aligned}
i \mathcal{I}= & -\frac{1}{2} \frac{\pi^{2}}{G_{0} G_{1} G_{2}} \frac{2 i}{\pi}\left(u_{1} \oint_{\mathcal{C}_{1}} d y_{0}\left(G_{1} \oint_{\mathcal{C}_{2}} d x_{1} \partial_{2}^{y} f(x, y)-G_{2} \oint_{\mathcal{C}_{2}} d x_{2} \partial_{1}^{y} f(x, y)\right)\right. \\
& +\frac{m^{*}}{\delta} \oint_{\mathcal{C}_{1}} d y_{1}\left(G_{2} \oint_{\mathcal{C}_{2}} d x_{2} \partial_{0}^{y} f(x, y)-G_{0} \oint_{\mathcal{C}_{2}} d x_{0} \partial_{2}^{y} f(x, y)\right) \\
& \left.+\frac{m^{*}}{\delta} \oint_{\mathcal{C}_{1}} d y_{2}\left(G_{0} \oint_{\mathcal{C}_{2}} d x_{0} \partial_{1}^{y} f(x, y)-G_{1} \oint_{\mathcal{C}_{2}} d x_{1} \partial_{0}^{y} f(x, y)\right)\right)
\end{aligned}
$$

Applying Stokes' formula to transform the loop integral over $\mathcal{C}_{1}$ into a surface integral over a disk $D_{1}$ such that $\partial D_{1}=\mathcal{C}_{1}$, and noting that $\oint_{\mathcal{C}_{2}} d x_{\mu} \partial_{\mu}^{x} h(x)=0$, we get

$$
\begin{aligned}
i \mathcal{I}= & -\frac{1}{2} \frac{\pi^{2}}{G_{0} G_{1} G_{2}} \frac{2 i}{\pi}\left(\int_{D_{1}} d y_{1} \wedge d y_{2} \oint_{\mathcal{C}_{2}} d x_{0} \frac{m^{*}}{\delta} G_{0} \int \frac{d^{3} k}{(2 \pi)^{3}}\left(-k_{1}^{2}-k_{2}^{2}\right)\left(\sum_{\lambda} \frac{k_{\lambda}^{2}}{G_{\lambda}}\right)^{-1} e^{-i(x-y) \cdot k}\right. \\
& +\int_{D_{1}} d y_{0} \wedge d y_{1} \oint_{\mathcal{C}_{2}} d x_{2} G_{2} \int \frac{d^{3} k}{(2 \pi)^{3}}\left(-u_{1} k_{1}^{2}-\frac{m^{*}}{\delta} k_{0}^{2}\right)\left(\sum_{\lambda} \frac{k_{\lambda}^{2}}{G_{\lambda}}\right)^{-1} e^{-i(x-y) \cdot k} \\
& \left.+\int_{D_{1}} d y_{2} \wedge d y_{0} \oint_{\mathcal{C}_{2}} d x_{1} G_{1} \int \frac{d^{3} k}{(2 \pi)^{3}}\left(-\frac{m^{*}}{\delta} k_{0}^{2}-u_{1} k_{2}^{2}\right)\left(\sum_{\lambda} \frac{k_{\lambda}^{2}}{G_{\lambda}}\right)^{-1} e^{-i(x-y) \cdot k}\right)
\end{aligned}
$$

Given that $G_{1}=G_{2}$, Eq. B6 is greatly reduced if $\mathcal{C}_{1}$ lies within a spatial plane,

$$
\begin{aligned}
\mathcal{I} & =-\frac{1}{2} \frac{\pi^{2}}{G_{0} G_{1} G_{2}} \frac{2}{\pi} \int_{D_{1}} d y_{1} \wedge d y_{2} \oint_{\mathcal{C}_{2}} d x_{0} \frac{m^{*}}{\delta} G_{0} \int \frac{d^{3} k}{(2 \pi)^{3}}\left(-k_{1}^{2}-k_{2}^{2}-\frac{G_{1}}{G_{0}} k_{0}^{2}\right)\left(\sum_{\lambda} \frac{k_{\lambda}^{2}}{G_{\lambda}}\right)^{-1} e^{-i(x-y) \cdot k} \\
& =\frac{1}{2} \frac{\pi^{2}}{G_{0} G_{1} G_{2}} \frac{2}{\pi} \frac{m^{*}}{\delta} G_{0} G_{1} \int_{D_{1}} d y_{1} \wedge d y_{2} \oint_{\mathcal{C}_{2}} d x_{0} \delta^{(3)}(x-y) \\
& =\left(\lambda_{0} / \lambda_{p}\right)^{2} \pi \Theta
\end{aligned}
$$


in which $\Theta$ is the Gaussian linking number of $\mathcal{C}_{1}$ and $\mathcal{C}_{2}$ loops. To obtain the last line of above identity, the definition of $\lambda_{p}$ and $\lambda_{0}$ in Eq. 12 is applied. 